\title{
Investigation of Structural Transformations During the Manufacturing of Expanded Snacks for Reformulation Purposes
}

\author{
R. G. M. van der Sman ${ }^{1}$ (D) . J. Williams ${ }^{2}$. J. R. Bows ${ }^{2}$ \\ Received: 8 March 2020 / Accepted: 17 September 2020 / Published online: 10 October 2020 \\ (C) The Author(s) 2020
}

\begin{abstract}
In this paper, we investigate the functionality of potato-based ingredients present in indirectly expanded snacks via careful analysis of their transformation during processing. This research is driven by the desire of industry to develop similar snacks for upcoming markets, where the potato-based ingredients are replaced by other starch sources, which are locally available and at a lower cost. For a range of reformulated snacks, the transformations of starchy ingredients are analysed with a wide variety of experimental methods, like DSC, XRD, and XRT. Our analysis shows that ingredients undergo little transformations during extrusion, which is indeed intended to be mild. During frying native tuber starches (potato and tapioca starch) fully gelatinize, while cereal starches show little gelatinization and swelling. Despite the gelatinization of tuber starches, the particulate character of ingredients is retained. Replacement of pregelatinized potato starch with other starches shows little change in structure. The evolution of the structure of the reformulated snacks are analysed with the CDS formalism. We conclude that gel formers and hard fillers present in the analysed formulations had little functionality regarding texture or structure. For texture, it appears to be required that the matrix composes of a bicontinuous structure of soft fillers, namely gelatinized tuber starches and potato dehydrates. Both these ingredients can be replaced by other tuberstarch sources if the aggregation of the two soft fillers can be prevented. Commercial availability of tuber flours can still be an issue.
\end{abstract}

Keywords Expanded snacks · Ingredient functionality · Reformulation

\section{Introduction}

Potato-based snacks are very popular in the Western world. Most of the potato-based snacks are made via indirect expansion. Using a single screw extruder at low temperature, ingredients are mixed and shaped into a halfproduct (a pellet), which is dried for long shelf-life stability [1]. This cold extrusion is intended to be mild, without starch gelatinization, similar to pasta processing [3, 33]. Pellets are expanded via frying them in hot oil. Often,

Electronic supplementary material The online version of this article (https://doi.org/10.1007/s11483-020-09652-w) contains supplementary material, which is available to authorized users.

$\triangle$ R. G. M. van der Sman

ruud.vandersman@wur.nl

1 Wageningen Food Biobased Research Wageningen University \& Research, Wageningen, Netherlands

2 PepsiCo R\&D, Leicester, UK these snacks have been developed in an artisanal way, while lacking the explicit knowledge of the ingredient functionality regarding structure and texture [26, 27].

This lack of knowledge poses problems for the industry for producing similar indirectly expanded snacks available for upcoming markets like India, where potato-based ingredients are often not locally available. Hence, the industry is engaged in a research program to replace potatobased ingredients with locally available low-cost starch resources [25, 32]. In particular, there will be a challenge to replace the so-called potato dehydrates, which is the industrial name for the group of ingredients based on cooked potato cells, which can be either potato granulates (intact cells) and potato flakes, which are drum-dried cells which have lost the integrity of the cell [27]. These ingredients are based on starch encapsulated in a cell wall matrix. A similar group of ingredients, based on another botanical source (other than potato) is not commercially available, as far as we know.

A scan of food science literature shows that there is little research performed on how certain starches sources can be 
substituted with other sources. We are aware of only a single study on starch substitution in fish crackers (keropok) [17]. We conclude that to be able to replace the potato-based starch ingredients, one has to have good knowledge of the functionality of these ingredients.

Starchy ingredients can undergo transformations during extrusion processing such as swelling, gelatinization/melting, leaching of amylose, and retrogradation. All these transformations can have a large impact on the ingredient functionality regarding the structuring. Hence, it is important to have good knowledge of the transitions of each ingredient during processing. For a precise description of the ingredient functionality and their transformations, we will make use of the Complex Dispersed Systems (CDS) notation [23], which we have applied earlier to describe the structuring of potato-based snacks [27], and sweet biscuits [29].

For the ingredient functionality, we will build upon earlier investigations: 1) the initial study towards ingredient functionality in extruded food materials is by [8], 2) the classification of swelling behaviour of different starch sources [4], 3) our earlier review papers [25, 27], and 4) our recent research paper on filler ingredient functionality [32].

Guy has distinguished three main classes of ingredients [8]: a) gel formers, b) matrix formers, and c) fillers. Guy has considered also several minor ingredients, but we regard that these ingredients do not have much functionality regarding the structuring of the snacks. Matrix formers are polymeric materials, like (gelatinized) starch, that constitutes the continuous phase, but can not form a gel by themselves. Gelling functionality is provided by a minor polymeric ingredient, such as amylose. Fillers are considered as a dispersed phase, that retains a particulate character. We have adopted this classification of ingredients in our earlier review paper [27].

However, our previous work [32] has shown that we have to subdivide the class of fillers into soft and hard fillers, because of their different functionality. Hard fillers like rice flour and cereal starches do not gelatinize during either extrusion or frying, due to restricted swelling by proteins and lipids present in the starch granule [4]. During frying the hard fillers promote bubble coalescence and premature bubble rupture, leading to thick bubble walls. Soft filler functionality can be delivered by 1) tuber starch granules (like potato and tapioca) and 2) potato dehydrates, i.e. potato granulates (intact cooked potato cells) and potato flakes (drum dried potato cells, with partially ruptured cell walls) [32]. The tuber starches will gelatinize during frying and then render soft filler functionality. As raw ingredient potato dehydrates already contain pregelatinized starch, encapsulated in cell wall material, and hence they will also behave as soft fillers. Native starches are assumed to promote bubble nucleation, which is thought to start in the hilum of the granule [6]. Soft fillers will also promote cell opening during expansion (allowing steam to escape) [32]. Products with attractive crispiness are characterized by a mixture of both soft and hard fillers [32].

Gel formers provide cohesiveness to the dough, via forming a network embedding the fillers and matrix formers. Ingredients used as gel former are pregelatinized starches, or amylose leached from potato flakes [15, 27]. Because of the large amounts of tuber starches and potato dehydrates present in the formulations [32], these ingredients are also thought to have both matrix former as soft filler functionality [27].

In this study, we have performed an extensive experimental program to elucidate further the functionality of potato-derived ingredients in the expanded snacks, and how this functionality can also be achieved with non-potato starch sources. The paper is organized as follows. First, we present the initial research questions regarding ingredient functionality in potato-based snacks, and their replacement by alternative starchy ingredients. Subsequently, the experimental design is given via which we have investigated the research questions, via observing the transformations of these ingredients during processing. After presenting the basic results, we conclude with the discussion of the results and research questions.

\section{Materials and Methods}

\section{Ingredients and formulations}

Snack formulations consist of mixtures of ingredients from the above-defined classes. Except for the hard filler, the other functional ingredients can be derived from potato. In this research, we will investigate whether the potato-based ingredients can be obtained from other botanical sources like cereals and other tubers, and that are commercially available. Only, non-potato ingredients similar to potato dehydrates are not commercially available. Consequently, we intend to mimic them via a mixture of potato fibers and pregelatinized starch, from potato or tapioca. Tapioca has slightly smaller granules than potato, and it has significantly less phosphate, which leads to somewhat reduced swelling in excess water. But we expect that this difference in swelling is not important at the reduced water content of the snack pellets.

In the formulations we have used potato dehydrates as soft fillers, cereal starches as hard fillers, native tuber starch granules as matrix formers, and pre-gelatinized starches as gel formers. As indicated by [4], cereal starches have restricted swelling capacity due to proteins and lipids present inside the starch granules. Consequently, it is found that cereal starches present in snack pellets do not gelatinize or swell after frying [17, 21, 25, 32]. 
Table 1 List of used ingredients with functionality and detailed structure in CDS

\begin{tabular}{|c|c|c|c|c|c|}
\hline Code & Ingredient & Supplier & Trade Name & Functionality & Structure \\
\hline & Soft filler & & & & \\
\hline PPG & Cooked Potato Granulates & Emsland & Emgranule 3364 & $\mathcal{C}$ & $\left(A P / A M_{x}\right) @ C$ \\
\hline PFL & Cooked Potato Flakes & Emsland & Emflake 3916 & $\mathcal{C} / G$ & $(C+A P) / A M_{x}$ \\
\hline \multirow[t]{2}{*}{ PFB } & Potato Fibers & KMC & Fiberbind 300 & $\mathcal{C}$ & C \\
\hline & Hard filler & & & & \\
\hline NRF & Native rice flour & Fen Srl & - & $\mathcal{F}$ & $\left(A M / A P_{x}\right) @ P$ \\
\hline NCS & Native corn starch & Fen Srl & - & $\mathcal{F}$ & $\left(A M / A P_{x}\right) @ P$ \\
\hline \multirow[t]{2}{*}{ HAC } & Hi-amylose corn starch & Ingredion & Hylon VII & $\mathcal{F}$ & $A M_{x} @ P$ \\
\hline & Matrix former & & & & \\
\hline NPS & Native Potato Starch & Emsland & - & $\mathcal{S}_{x}$ & $A M / A P_{x}$ \\
\hline NTS & Native Tapioca Starch & Fen Srl & - & $\mathcal{S}_{x}$ & $A M / A P_{x}$ \\
\hline \multirow[t]{2}{*}{ WPS } & Waxy Potato Starch & Avebe & Eliane 100 & $\mathcal{S}_{x}$ & $A P_{x}$ \\
\hline & Gel former & & & & \\
\hline GPS & Pre-gelatinized potato starch & KMC & Coldswell 1111 & $\mathcal{S}_{g} / \mathcal{G}$ & $A P_{g} / A M$ \\
\hline GTS & Pre-gelatinized tapioca starch & KMC & Coldswell 1112 & $\mathcal{S}_{g} / \mathcal{G}$ & $A P_{g} / A M$ \\
\hline GRF & Pre-gelatinized rice flour & S\&B Herba & Parboiled rice flour & $\mathcal{S}_{g} / \mathcal{G}$ & $\left(A P_{g} / A M\right) @ P_{g}$ \\
\hline
\end{tabular}

The actual ingredients used in our experiments are listed in Table 1, where they are sorted according to the defined classes. Furthermore, we have listed their suppliers and trade names. The table also lists the composition and structure of these ingredients, which are described in the CDS formalism. This formalism is explained in detail below.

The investigated formulations are listed in Table 2, where we have listed the weight percentage of each ingredient present. We use two commercial formulations $R_{1}$ and $R_{2}$ as references, which contain predominantly potato-based ingredients. In the reformulations, we will keep the ratios between functional ingredients the same as in the reference $R_{1}$.
To obtain insight into the structuring functionality of the used ingredients, we will partially replace the starchy ingredients of the references with other ingredients, which can also be taken from other botanical sources to learn how to replace potato-based ingredients with other starches. To focus this research, the reformulations are based on particular research questions, that are based on hypotheses and assumptions concerning ingredient functionality in the reference formulations. These research questions are given below.

In Table 2 all starchy ingredients add up to $100 \%$. On top of the starchy ingredients, all formulations will contain additional salt (1\%) for taste and monosodium glyceride

Table 2 Starchy ingredients in snack formulations given in weight percentage of total starch ingredients

\begin{tabular}{|c|c|c|c|c|c|c|c|c|c|c|}
\hline Coding & $R_{1}$ & $R_{2}$ & Cell1 & Cell2 & Cell3 & Cell4 & Filler1 & Filler2 & Filler3 & Filler4 \\
\hline Research Question & 0 & 0 & 1,2 & 1 & 1,4 & $1,3,4$ & 5,7 & 5,6 & 5,6 & 5,7 \\
\hline PPG & 46 & & & 22 & & & 45 & 45 & 45 & 45 \\
\hline PFL & & 30 & & & 45 & & & & & \\
\hline PFB & & & 8 & 4 & & 8 & & & & \\
\hline NRF & 8 & 8 & 8 & 8 & 8 & 8 & & & & \\
\hline NCS & & & & & & & & & 8 & \\
\hline HAC & & & & & & & & 8 & & \\
\hline NPS & 45 & 52 & 47 & 45 & & & 46 & 36 & 46 & 46 \\
\hline NTS & & 9 & & & 46 & 46 & & & & \\
\hline WPS & & & & & & & & 10 & & 6 \\
\hline GPS & & & 36 & 20 & & & 2 & & & 2 \\
\hline GTS & & & & & & 37 & & & & \\
\hline GRF & & & & & & & 6 & & & \\
\hline
\end{tabular}


$(0.1 \%)$, as a processing aid. The moisture content of the dough will be around $25-35 \%$, based on total weight.

\section{Snack Manufacturing}

Commercial snacks are made with the following processing steps: 1) mixing of dry ingredients with water, 2) cold extrusion, 3) drying, 4) equilibration, and 5) frying in oil. Following these processing steps, the snacks are produced at the pilot plant of Fen Srl (Italy).

For the mixing, a large ribbon blender is used producing batches of $30 \mathrm{~kg}$. First, the raw dry ingredients are mixed for 10 minutes, and subsequently, cold water is added, while continuing to mix for at least 10 minutes. The aim is to obtain a mix with a total moisture content of about $34 \%$. However, the raw ingredients have a moisture content of about $14 \%$. The amount of water to be added is based on the measured moisture content of the raw ingredients, and their ratios in the formulations. The measured final moisture content of the hydrated mixes was in the range of 33-36\%. After mixing the wet dough is held for equilibration for at least 30 minutes. Subsequently, the mixed ingredients are fed into the extruder via a feeding screw.

The extruder has four sections, with consecutive temperatures of $40,70,70$, and $50^{\circ} \mathrm{C}$. The feeding screw has a RPM of 27, and the cooking screw has a RPM of 42, rendering throughput of $38-48 \mathrm{~kg} / \mathrm{h}$. The die pressure was in the range of $12-16 \mathrm{MPa}$, and the die temperature was in the range $50-60^{\circ} \mathrm{C}$. Square tubes are formed via a die with square holes having a width $5.5 \mathrm{~mm}$, and slit size 0.55 $\mathrm{mm}$. One sample (labeled as Cell3b) has also been extruded at higher temperatures due to the bad performance of the extruder. The applied settings were $40,85,85,65^{\circ} \mathrm{C}$. Strands of the extrudate are cut via a free-standing cutting system. This way of cutting may introduce variation in the length of the pellets, and also some deformation of the cross-section.

After extrusion, the samples are air dried with a bed dryer for at least 50 minutes. Subsequently, they are equilibrated to ambient air for 24-36 hours, resulting in final moisture contents in the range of $12-12.5 \%$. After drying the pellets are fried at $180^{\circ} \mathrm{C}$, for about 2 minutes. After the end of the bubbling phase, the expanded snacks will float on the surface of the oil, which the sign that they can be taken out of the frying oil.

\section{Experimental Methods}

\section{Moisture content and water activity}

The moisture content of the samples is analysed using a Mettler Toledo halogen moisture analyser HB 43, which is set to $105^{\circ} \mathrm{C}$. Their water activity is also determined with a Novasina AW-Sprint TH500 water activity meter.

\section{XRD Method}

Wide-angle X-ray scattering powder diffraction (XRD) patterns were recorded on a Philips PC-APD diffractometer (Model PW 3710) in the reflection geometry in the angular range $7-40^{\circ}(2 \theta)$, with a step size of $0.02^{\circ}(2 \theta)$ and an acquisition time of $4.0 \mathrm{~s}$ per step. The $\mathrm{Cu} \mathrm{K} \alpha 1$ radiation $(\lambda=0.1542 \mathrm{~nm})$ from the anode, generated at $30 \mathrm{kV}$ and $50 \mathrm{~mA}$, was monochromatized using a $15 \mu \mathrm{m}$ Ni foil. The diffractometer was equipped with a $1^{\circ}$ divergence slit, a 0.2 $\mathrm{mm}$ receiving slit, and a $1^{o}$ scatter slit.

Raw ingredients were measured in their powdery form as obtained from the manufacturer. The pellets were cryogenically milled in a Retsch mill (pore size of sieve: $0.5 \mathrm{~mm}$ ) at $1500 \mathrm{rpm}$ for less than 1 minute. Due to their adherence to the sieve, it was decided to grind the expanded snacks with a mortar and a pestle.

\section{DSC method}

Differential Scanning Calorimetry (DSC) is applied with a TA Instruments Q20 machine. Samples are put in aluminum cups of $60 \mu \mathrm{l}$. Samples are heated from 10 to $100^{\circ} \mathrm{C}$ at a rate of $10 \mathrm{~K} / \mathrm{min}$. The cups have received samples of $30-$ $40 \mathrm{mg}$. To the pellets and expanded snacks, we have not applied milling e.g. The snack products are broken manually and a fractured piece is taken of sufficient weight, and that also fits in the DSC cup. Also, extra measurements are performed on hydrated samples, where extra water is added such that dry matter/water ratio is $1: 1$.

\section{PSD after hydrolysis}

To determine the amount of fillers present in the sample we use the same PSD (Particle Size Distribution) method as in our previous paper [32]. First, we hydrolyse the amorphous starch in the samples via enzymes. The particle size distribution of the hydrolysed suspension is determined. To determine the relative amount of fillers in the dry material, the fillers are separated from the suspension via centrifugation. The relative amount is determined via weight measurement of the initial material and the centrifuged pellet.

The enzymatic hydrolysis is performed with barley $\beta$ amylase (A7130 from Sigma Aldrich), using an acetate buffer of $100 \mathrm{mM}$ sodium acetate and a $\mathrm{pH}$ of 5.2. First, we have dispersed about 2 grams of samples in $50 \mathrm{ml}$ of acetate buffer, including 2\% ethanol. The liquid is held at 35 degrees Celsius. Dispersion is performed mechanically via a magnetic stirrer for 30 minutes.

The enzyme is added to hydrolyse amorphous starch. We will use $\beta$-amylase, which is an exo-enzyme. It will make incisions at the end of the amylose and amylopectin chains. 
We expect that this enzyme has trouble entering/absorbing to the (partially) intact starch granules or potato cells [7, 19], also given the limited amount of time given for hydrolysis. We assume only soluble starch is hydrolyzed.

We take $5 \mathrm{ml}$ of the dispersion, and dissolve $12.5 \mathrm{mg}$ of enzyme in it, which is diluted with $25 \mathrm{ml}$ of buffer solution. Enzymatic hydrolysis is allowed at 35 degrees for 2 hours, while keep stirring. $6 \mathrm{ml}$ of the hydrolysed suspension is used for determination of the particle size distribution and microscopy.

The remaining suspension is centrifuged for 10 minutes at $18^{\circ} \mathrm{C}$ and $9000 \mathrm{~g}$. The supernatant is decanted, and the pellet is freeze-dried, and subsequently, the weight of the dry matter is determined. Knowing the initial weight of the samples, and their moisture content, we can determine the relative amount of fillers in the total dry weight of the material.

Particle size distribution is determined via a Malvern 2000. Furthermore, images are made of the hydrolysed suspension via dark-field microscopy, which enhances the contrast in unstained samples. Light originates only from scattering, and not from the directly transmitted light. This enhances the imaging of fibers and cell walls.

\section{XRT method}

For the X-ray tomography (XRT) we have used a GE Phoenix imager (General Electric, Wunstorf, Germany). The system contains two X-ray sources. The $180 \mathrm{kV}$ nano focus tube with a tungsten target is employed. $\mathrm{X}$-rays were produced with a voltage of $80 \mathrm{kV}$ and a current of 300 $\mu \mathrm{A}$. The images are recorded by a GE DXR detector array with 2024 x 2024 pixels (pixel size $200 \mu \mathrm{m}$ ). The detector is located $815 \mathrm{~mm}$ from the X-ray source. The object is placed $10.08 \mathrm{~mm}$ for expanded snacks and 30.41 $\mathrm{mm}$ for snack pellets from the X-ray source. This results in a spatial resolution of $2.5 \mu \mathrm{m}$ and $7.5 \mu \mathrm{m}$ respectively. A full scan consists of 1800 projections over $360^{\circ}$. The saved projection is obtained over $500 \mathrm{~ms}$ exposure time. GE reconstruction software (Wunstorf, Germany) is used to calculate the 3D structure via back projection. Further data analysis is performed with Avizo software version 9.2.0.

XRT imaging is performed on a small piece of the expanded snack, as shown in Fig. 1. Note, there are pores at the top and bottom, which have a direct connection to outside. These pores are excluded from data analysis for the pore size and wall thickness distribution. Some cells are filled with fat, which can be distinguished from the dry starch matter, via the greyscale intensity. Via counting pixels with fat, the fat volume fraction is computed. Subsequently, these cells are further treated as pores and contribute to the pore size. Before the image analysis, the raw XRT data is filtered to remove noise. The image is binarized via a certain

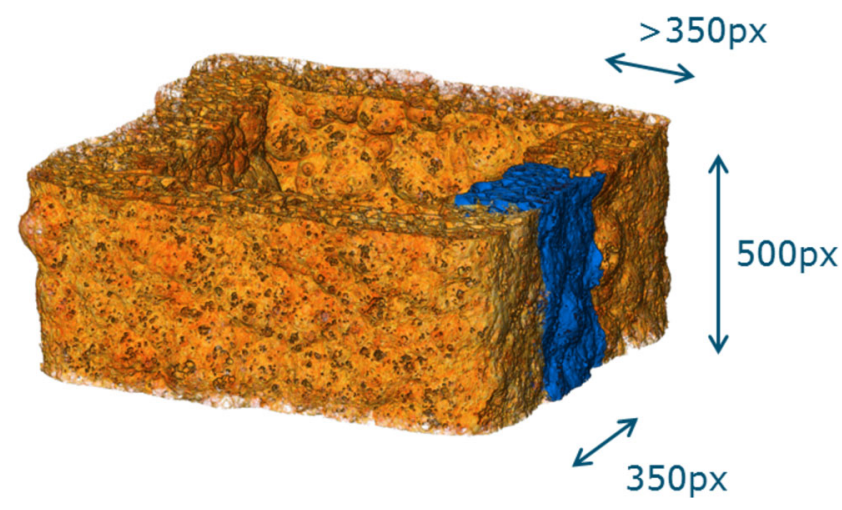

Fig. 1 Location where XRT quantitative analysis is applied

threshold. Via granulometry using Avizo, the pore size and wall thickness distributions are computed. The details of the image analysis algorithms can be found in our previous publication [32].

\section{Sensorial evaluation}

The expanded snacks are sensorial evaluated using an untrained, but experienced panel of PepsiCo employees. The panel consisted of 6 people, which are asked to score the expanded snacks on several attributes, using a 15-point scale, using the $R_{1}$ formulation as a reference (which has score 0 for all attributes). The scored attributes are: the amount of expansion, the appearance of blisters, the colour, flavour, hardness, crunchiness, crispiness, gumminess, and the ease of breakdown during eating.

\section{CDS Formalism}

\section{Ingredients structure}

We will describe the ingredients and their transformations using the Complex Dispersed System (CDS) formalism [23], which has already shown its value in describing the functionality of ingredients in potato-based expanded snacks [27], but also in biscuits [29] and cakes [30]. With CDS the structure of the system will be indicated by several symbols, extending the notation commonly used for emulsions, where O/W means oil dispersed into water. As in soft matter science we use a broad definition of the concept of dispersions, involving not only emulsions or liquid foams, but also solid foams like snacks, and solid-solid dispersions like chocolate [31].

Hence, ' $l$ ' can be generalized to denote a dispersion such as $D / C$, with $D$ the dispersed phase, and $C$ the continuous phase. $C$ or $D$ can be any food material, like water $(W)$, oil $(O)$, carbohydrate $(C)$, starch $(S)$, amylose $(A M)$, amylopectin $(A P)$, cell wall material $(C)$ proteins $(P)$, (water) vapour $(V)$, and air $(A)$. 
Materials that are at the interface between different phases are indicated with the '@' symbol, meaning 'stabilized by' or 'enclosed by'. $(O @ Z) / W$, means oil droplets stabilized by emulsifier $(Z)$ dispersed into water. Several materials are soluble in a liquid phase, for which we will use '+', meaning 'mixed with'. Hence, $W+$ $C$ represents a sugar solution. Via subscripts, we will occasionally indicate the physical state of ingredients. Native starch is semi-crystalline and is indicated by $S_{x}$. Amorphous starch in the glassy state is indicated with $S_{g}$. If the ingredients are in the rubbery state, the subscript is dropped.

All ingredients used in this research are listed in Table 1, where we have also described their presumed functionality and detailed structure using CDS. For the functionality we have used the new symbols to represent (functional) classes of materials, such as cell wall material (fibers) $(\mathcal{C})$, hard fillers $(\mathcal{F})$, gel formers $(\mathcal{G})$, and soft fillers $\mathcal{S}$. Note, in this particular study the gel former functionality is only provided by amylose $\mathbf{A M}$, provided by pregelatinized starches or cooked potato flakes. In the detailed structure of the dry ingredients, we have distinguished individual materials making up the composite ingredients.

\section{Transformations during processing}

To design our experiments, we have expanded our earlier hypotheses on how potato-based ingredients transform during processing and their role in building the structure. Our first hypotheses have been stated in our review paper [27], which have been formulated in the CDS formalism. In the review paper, we have not made a distinction between soft and hard fillers. Hence, we have expanded our first hypotheses, while distinguishing soft and hard fillers, which are shown in Table 3. We have denoted the ingredients as $G$ for gel formers, $C$ for potato dehydrates, $F$ for hard fillers, and $S$ for starches. For the starch, we will use indices to indicate its physical state ( $x$ for the crystalline state, and $g$ for the glassy state).

There are structural changes during the following (processing) steps: 1) mixing via cold extrusion, 2)

Table 3 Evolution of structure during processing of potato snacks described with CDS formalism

\begin{tabular}{ll}
\hline Step & Structure in CDS \\
\hline Dry mix & $\mathcal{W}+\mathcal{G}+\mathcal{F}+\mathcal{C}+\mathcal{S}_{x}$ \\
Mixing/Extrusion & $\rightarrow\left(\mathcal{F}+\mathcal{W} / \mathcal{C}+\mathcal{S}_{x}\right) / \mathcal{G}$ \\
Gelatinization & $\rightarrow(F+W / C+W / S) / G$ \\
Nucleation & $\rightarrow(F+W / C+V / S) / G$ \\
Expansion & $\rightarrow \mathcal{V} /(F+C+S) / G$ \\
Setting & $\rightarrow \mathcal{A} /\left(\mathcal{F}+\mathcal{C}+\mathcal{S}_{g}\right) / \mathcal{G}$ \\
\hline
\end{tabular}

gelatinization, 3) nucleation, 4) expansion, and 5) setting. Steps 2) to 4) are all happening during frying. After extrusion, the pellet is dried, but there is no change in the structure during this processing step.

During extrusion mixing the dry ingredients are mixed with water. The ingredients before mixing are denoted as $\mathcal{W}+\mathcal{G}+\mathcal{F}+\mathcal{C}+\mathcal{S}_{x}$. During mixing the water is largely taken up by the pre-gelatinized starch present in the potato dehydrates. The swollen potato dehydrate is denoted as $\mathcal{W} / C$. During extrusion the fillers $\mathcal{F}$ and native tuber starches $\mathcal{S}_{x}$ do not gelatinize [32]. The pre-gelatinized starch added as gel former is assumed to build a network in which the other particulate ingredients are dispersed. Hence, the structure of the pellets exiting the extruder is $\left(\mathcal{F}+\mathcal{W} / \mathcal{C}+\mathcal{S}_{x}\right) / \mathcal{G}$

During frying there is first a temperature rise, putting ingredients into the rubbery state, and allowing the starch to gelatinize: $(F+W / C+W / S) / G$. We assume that some of the water from the potato dehydrate is taken up by the gelatinized tuber starch. Once, the temperature is at the boiling temperature steam bubble will form. [28]. We assume that these steam bubbles nucleate in the hylum of the gelatinized tuber starches [6]: $(F+W / C+V / S) / G$.

Subsequently, the nucleated bubble will expand due to the migration of water from the matrix, and its conversion to vapour $(\mathcal{V})$ [28]. The bubble is assumed to be dispersed in the matrix consisting of the gelatinized starches and the filler particles, entrapped in the gel network $: \mathcal{V} /(\mathcal{F}+\mathcal{C}+\mathcal{S}) / \mathcal{G}$

The hard fillers present in the bubble wall will provide the partial rupture of the expanding bubble walls [32]. Consequently, the steam can escape the expanded snack, which is replaced by air: $\mathcal{A} /(F+C+S) / G$.

After bubble rupture, the expanded snacks are taken out of the fryer, and they are cooled down. All amorphous ingredients enter the glassy state. The hard filler is assumed to remain inert: $\mathcal{A} /\left(\mathcal{F}+\mathcal{C}+\mathcal{S}_{g}\right) / \mathcal{G}$.

\section{Experimental design}

With the experimental program, we investigate the transformations of starchy ingredients during the various steps of their processing. We will study these transformations with a wide array of experimental methods, which are summarized in Table 4. In the table, we have also indicated to which samples the methods are applied, and what is actually determined. Next to this analysis of the ingredient transformations, we have also performed a basic analysis of the dimensions of expanded snack for quantifying expansion and sensorial evaluation with an untrained panel of PepsiCo employees for texture.

Via the experiments we will test the following research questions: 
Table 4 Experimental setup

\begin{tabular}{lll}
\hline Samples & method & Research question \\
\hline $\begin{array}{l}\text { Raw ingredients } \\
\text { Pellets }\end{array}$ & XRD & The type of crystallinity in pure ingredients \\
Expanded snacks & & $\begin{array}{l}\text { The degree of crystallinity after during extrusion } \\
\text { Amorphous state of the samples }\end{array}$ \\
Raw ingredients & DSC & Degree of gelatinization at low moisture in pure ingredients \\
Dry Pellets & & Occurrence of gelatinization during extrusion \\
Dry Expanded snacks & & Occurrence of gelatinization during frying \\
Wet Mix & & Degree of gelatinization (DG) at high moisture \\
Wet Pellets & & Impact of extrusion on DG (compared to dry ingredients) \\
Wet Expanded snacks & XRT & Impact of frying on DG (compared to pellets) \\
Expanded snacks & PSD & Quantification of structure related to texture \\
Wet Dough & & Amount of filler particles present in dry mix \\
Pellets & & Degradation of particles/fillers via extrusion \\
Expanded snacks & MC/ $a_{w}$ & Degradation of particles/fillers by frying \\
Raw ingredients & & Amount of water in amorphous fraction (dry) \\
Wet Dough & & Amount of water in amorphous fraction (wet) \\
Pellets & & Moisture absorbed by amorphous starch; what is $a_{w}$ \\
Expanded snacks & Water activity + moisture + fat content
\end{tabular}

1. Is it essential for the soft filler functionality of the cooked potato granulates (PPG) to have an intact cell wall encapsulating the gelatinized starch?

2. Can the functionality of potato flakes (PFL) be mimicked by potato fiber suspension (PFB) and pregelatinized potato starch (GPS) ?

3. Can pregelatinized potato starch (GPS) be replaced by pregelatinized tapioca starch (GTS) ?

4. Can native potato starch (NPS) be replaced by native tapioca starch (NTS) ?

5. Is it important for the texture to have hard fillers like native rice flour (NRF) present in the formulation ?

6. Is the size of the hard filler important for texture ?

7. Does native rice flour (NRF) also have functionality towards gel former?

With the new formulations Cell1-Cell4, we test Research Questions 1-4. In Cell1 we mimic the cooked potato granulates (PPG), with the mixture of PFB+GPS. In Cell2 we do a similar replacement, but only for $50 \%$. In Cell3 we replace potato granulates (PPG) with potato flakes (PFL), to check the soft filler functionality is only obtained if starch encapsulated by a cell wall. Cell4 is similar to Cell1, but native potato starch (NPS) and gelled potato starch (GPS) replaced by tapioca starches (NTS and GTS). Tapioca is a tuber starch similar to potato, which will also swell rapidly.

In formulations Filler1-Filler4 we will test Research Questions 5-7. In Filler1 there is no hard filler present, we have replaced NRF with GRF, which is pregelatinized. In Filler2 we have replaced NRF with HAC, which has both larger granule size and lower swelling capacity. Because of the high amylose content of HAC, we will replace some of the native potato starch (NPS) with waxy potato starch (WPS) to compensate for the high amylose content. In Filler3 we use NCS as hard filler, which is expected to have similar swelling properties as native rice flour (NRF). But, it has a larger size - that might impact texture (cell opening). In Filler4 we replaced the hard filler (NRF) with a soft filler, lacking amylose (WPS). Hence, we test whether rice granules only have the functionality of hard filler, or that they also contribute significantly to gel formation.

\section{Results}

\section{Dimensions, Appearance, and Moisture Content}

Images of all dry pellets are shown in Figure S.1 of the Supplementary Materials (S.M.). At standard extrusion conditions pellet Cell3 has shown bad mixing, and consequently, it is extruded again at higher temperatures (see Materials and Methods). These different samples are designated as Cell3a and Cell3b, with standard and high extrusion temperatures respectively. Both types of pellets have been fried up. The dimensions of the pellets are slightly different as shown in Table S.1 in the S.M. The table also shows a considerable deviation of the cross-section of the pellets from the square die (being 55 x $55 \mathrm{~mm}$ ). The change of cross-section can be attributed due to die 
Fig. 2 All expanded pellets

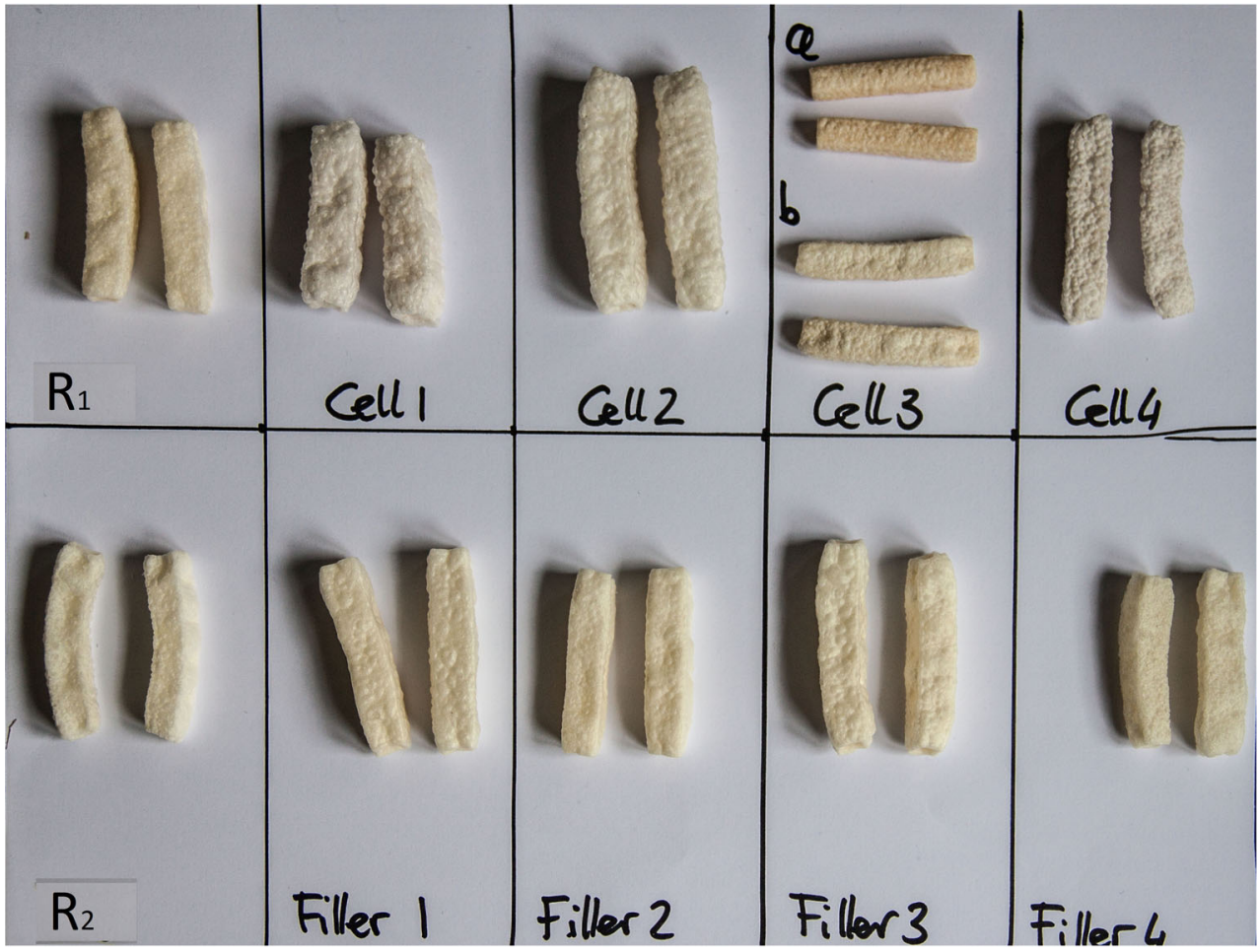

swell, deformation during cutting and drying. However, it is proven difficult to connect the pellet dimensions to properties of the expanded snacks. Hence, it will not be discussed any further.

In Fig. 2 we show images of all expanded snacks. From the images, one can immediately notice the differences in surface texture between the Cell formulations and the Filler formulations. Several Cell formulations have a bubbly surface. Also, there is considerable variation in dimensions amongst the expanded snacks, as shown in Table S.2 in the S.M. There, we also show the remaining moisture content and water activity $a_{w}$ of the expanded snacks.

The Table S. 2 shows that Filler, $R_{1}$, and $R_{2}$ formulations are reasonably comparable, with widths in the range of 8-9 mm, and $a_{w} \approx 0.05-0.07$. The Cell formulations can be divided into two groups. The Cell3a/b formulations show very little expansion, while the other Cell formulations (Cell1, Cell2, and Cell4) show large expansion, while the water activity has remained higher than that of the Fillers, with $0.11<a_{w}<0.15$.

\section{Sensorial Evaluation and Microscopy}

The evaluation of sensorial traits as scored by the PepsiCo expert panel is shown in Table 5.

The results show that we have obtained a wide variety of texture of the expanded snacks, albeit that the differences amongst the Filler formulations are smaller than amongst the Cell formulations. Moreover, the texture and crispiness of the Filler formulations are similar to the reference $R_{1}$.

Table 5 Sensorial evaluation of various texture parameters

\begin{tabular}{llllllllllll}
\hline Attribute & $R_{1}$ & $R_{2}$ & Cell1 & Cell2 & Cell3a & Cell3b & Cell4 & Filler1 & Filler2 & Filler3 & Filler4 \\
\hline Expansion & 0 & 1 & 3 & 3 & -3 & -2 & 1 & 0.5 & 0 & 0.5 \\
Blister & 0 & 0 & 2 & 1.5 & -2 & -1 & 1 & 0 & -0.5 & 0.5 & 0.5 \\
Colour & 0 & -0.5 & -3 & -2 & 2 & 1 & -3 & 0 & 0 & -0.5 & -0.5 \\
Flavour & 0 & -1 & -3 & -2 & -1 & 0 & -2 & 0 & 0 & -1 & -0.5 \\
Hardness & 0 & -0.5 & -3 & -2 & 3 & 2 & 2 & -0.5 & -1 & -1 & 0 \\
Crunchy & 0 & -0.5 & -3 & -2 & 3 & 2 & -2 & 0 & 0 & -1 \\
Crispy & 0 & -0.5 & -3 & -2 & -2 & -1 & -2 & 0 & 0 & -1 \\
Gummy & 0 & -0.5 & 2 & 1 & 1 & 0.5 & 1.5 & 0 & 0 & 0 \\
Breakdown & 0 & -1 & -2 & -2 & -2 & -2 & -2 & 0 & 0 & 0 \\
\hline
\end{tabular}


For the Cell formulations with NTS (Cell3a/b and Cell4) the texture is quite hard, and not crispy. The Cell formulations with a significant amount of gelled starch and potato granulate (Cell1 and Cell2) are soft, gummy less crispy than the reference $R_{1}$, while showing a large expansion (as follows from their dimensions in Table S.2).

With microscopy, we have investigated dry ingredients, pellets, and mixes, pellets, and expanded snacks after wetting. The images are shown in the Supplementary Material. The following remarkable observations, regarding dispersion and aggregation of several particulate ingredients, are made from the microscopy photos:

- Native tapioca starch (NTS) appears to aggregate, and particularly to potato cell walls (whether as cells (PPG), flakes (PFL), or fibers (PFB).

- $\quad$ Rice flour (NRF) disperses poorly, and shows large aggregates, even in the expanded snacks.

- $\quad$ Potato fibers (PFB) and possibly also flakes (PFL) tend to aggregate and trap particulates within them.
- Waxy potato starch is perhaps aggregating, these structures are observed only in Filler2 and Filler4. After the extrusion, the aggregation is absent.

\section{XRD}

First, we have analysed the raw materials such that we may identify them in the pellets and expanded snacks. These results are shown in Fig. 3. We have made the following grouping of results: a) gelatinized starches (top left), b) potato dehydrates (top right), c) native starches with Bcrystal (bottom left), and d) native starches with A-type (bottom right).

In the graphs, we have indicated the expected peaks for B (left) and A (right) crystal types, as dashed lines. Tuber starches like native potato starch (NPS and WPS) have a B-type signature, while cereal starches like native corn (NCS) and native rice flour (NRF) have an A-type signature. Tapioca starch (NTS) has a signature that is similar to Atype crystals, with only a single peak around angle $=22^{\circ}$.
Fig. 3 XRD results on the raw ingredients, in various groupings, as explained in the main text. In the top figures, the dashed lines indicate the spectrum from retrograded amylose, while in the bottom the dashed lines indicate either the B-type crystals (left) or the A-type crystals (right)
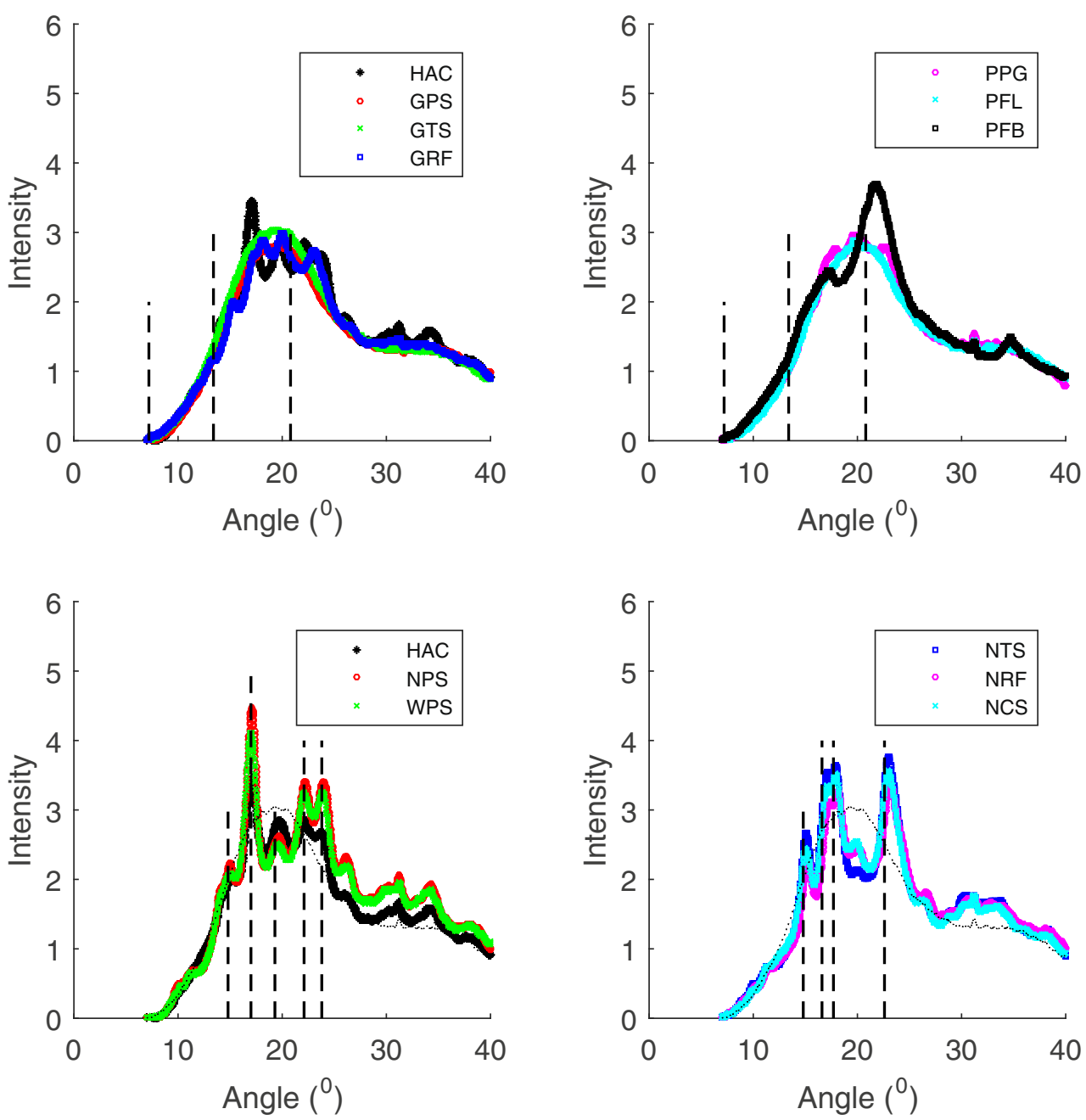
High-amylose corn (HAC) has a B-type crystal, as known from literature [12].

The pre-gelatinized potato and tapioca starch (GPS and GTS) and potato flakes (PFL) have a clear amorphous signal, while small peaks are observed for potato granules (PPG) and gelatinized rice flour (GRF). We have compared those peaks with the V-type crystal signature of retrograded amylose (dashed lines in the top figures), but we observe little correspondence with that. However, there is also little correspondence with A and B crystal type. The signal of GRF resembles that of HAC. Rice starch is relatively rich in amylose, which can explain the similarity of the signature. We assume the signature is due to some residual crystallinity in amylopectin, either due to retrogradation or incomplete (pre)gelatinization. The peak in the signature of potato fibers (PFB) is due to crystalline cellulose.

The XRD spectra of the pellets and the expanded snacks are shown in Fig. 4. We observe that all formulations still have crystalline starch. The spectra of all four Filler formulations are nearly indistinguishable. There are some differences between Filler formulations, and $R_{1}$ and $R_{2}$. There is more variation in the spectra of the Cell formulations. Cell3a/b and Cell4 show two peaks near $17^{\circ}$, and a single peak near $23.5^{\circ}$, which is the signature of the native tapioca starch, which has A-type crystal - while native potato starch has B-type crystals. The spectra of Cell1 and Cell2 are very similar, as can be expected from a similar amount of NPS.

From the spectra of the expanded snacks, we observe in general very little peaks, indicating that much of the crystalline starch has molten during frying. All Filler formulations give nearly indistinguishable spectra. We find more differences amongst the Cell formulations, but the differences are too small to draw any conclusions.

\section{Particle size distribution}

The results of the PSD analysis are shown in Fig. 5. The results of the hydrated mixes (sampled before extrusion) are shown at the top pane of Fig. 5. One can observe very distinct size distributions of the different ingredients. Via the differences in formulations, we can be traced back to these distributions to the individual ingredients. Hence, NTS has a diameter of about 20-30 $\mu \mathrm{m}$, NPS has a diameter of about
Fig. 4 XRD results on the extruded pellets (top) and the expanded snacks (bottom), grouped in Cell and Filler formulations. The dashed lines indicate the B-type crystals type, present in potato, and the dotted lines indicate the A-type crystals, present in cereals
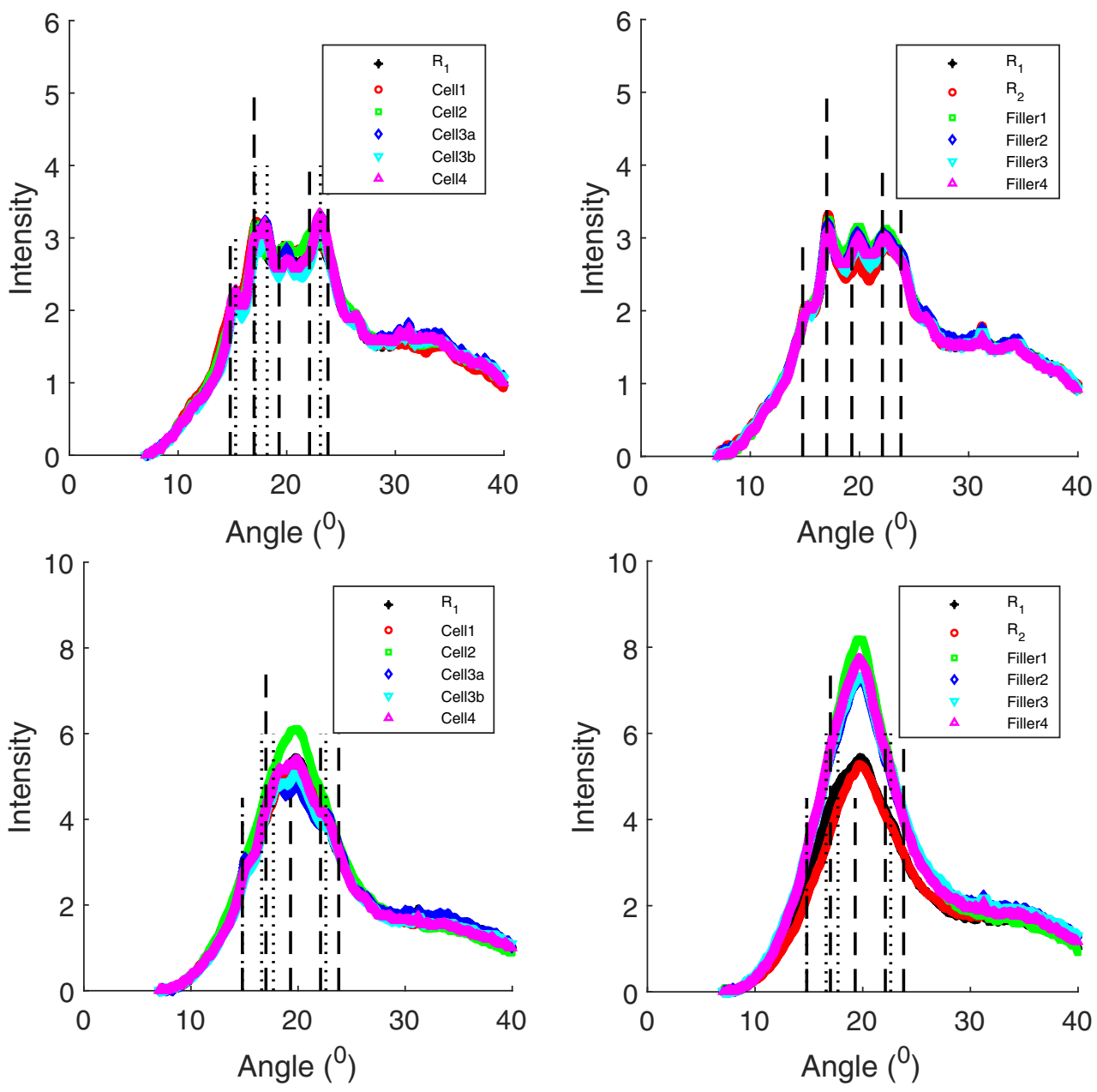
Fig. 5 PSD of a) hydrated mixes (top), extruded pellets (mid), and expanded snacks (bottom), grouped in Cell and Filler formulations
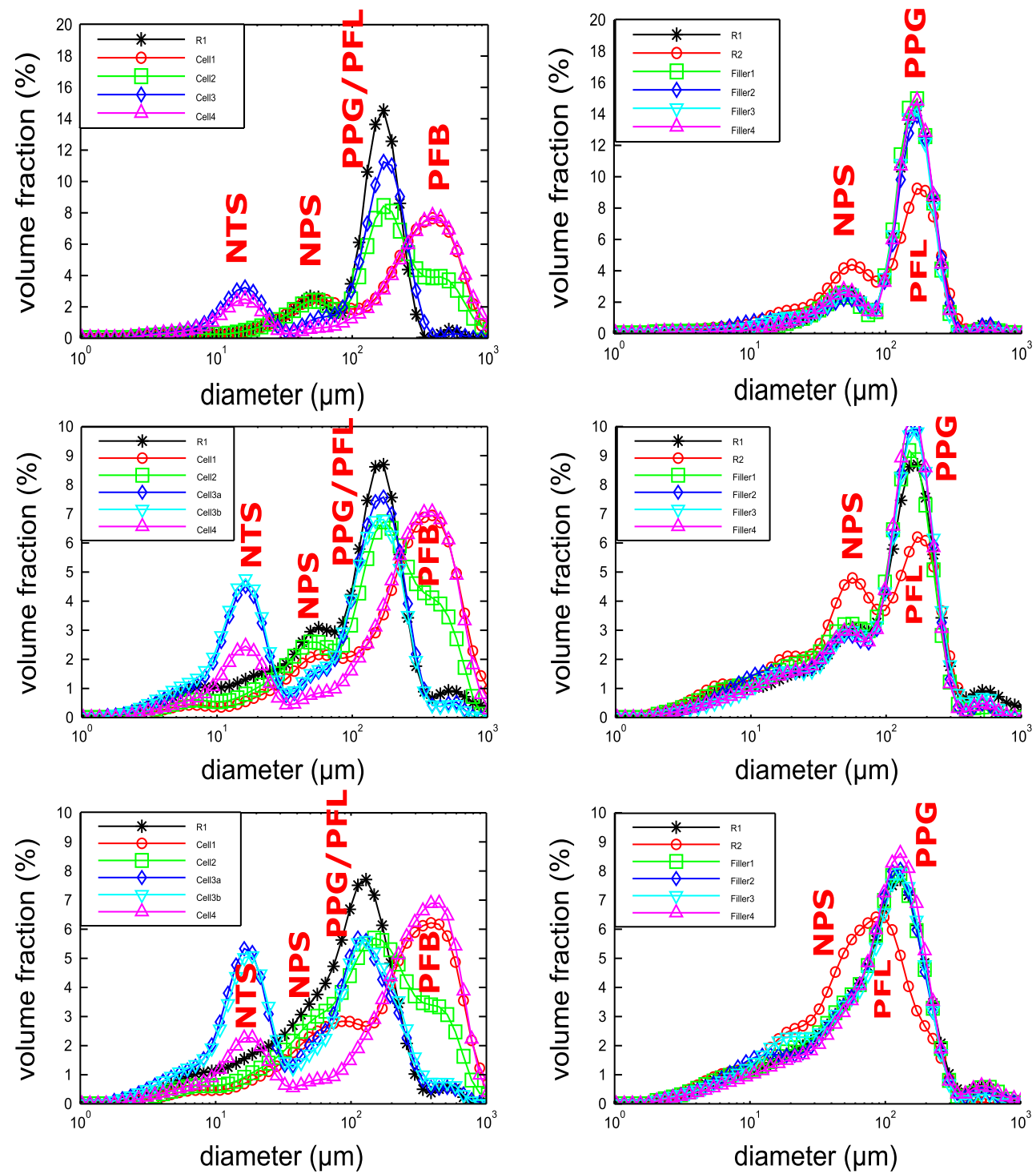

$80 \mu \mathrm{m}$, while PPG and PFL have a diameter of about 150 $\mu \mathrm{m}$, while PFB shows large aggregates of $500 \mu \mathrm{m}$. Other ingredients can not be distinguished because of their small amounts present in the formulations.

These different populations are still clearly present in the PSD of the pellets, as shown in the middle pane Fig. 5. But also, in the expanded snacks there are still distinguishable particles remaining, as shown in their PSD in the bottom pane of Fig. 5. While the XRD measurements have shown that the native starches have lost their crystallinity, the PSD show they have not lost their particulate integrity. Moreover, they are even only slightly swollen due to a limited amount of water.

The changes in the PSD during processing for each formulation are shown in Figures S.2-S.3 shown in the Supplementary Materials. We observe little variation amongst Filler formulations, which also compare well with the reference $R_{1}$. But, there are significant differences in the changes of the PSD during processing amongst the Cell formulations. The PSD of the Cell1, Cell2, and Cell4 show hardly any changes, while Cell3a and Cell3b show strong changes of the PSD due to dispersion of the aggregates of PFL and NTS, as one can notice from the increase of NTS particles after extrusion.

Concerning individual ingredients, we can state that only potato granulates (PPG) and native potato starch (NPS) change in size. There is a reduction in size for PPG, due to extrusion and dehydration during expansion and drying, while there is an increase in size for NPS due to swelling associated with the gelatinization during frying. 


\section{XRT}

The XRT results are illustrated with representative crosssections of the expanded snacks, as shown in Figs. 6 and 7. The XRT images have been analysed in quantitatively in terms of the distributions of pore size and bubble wall thickness, as shown in Figs. 8 and 9.

We observe distinct differences amongst the Cell formulations, while many of the Filler formulations are similar to reference $R_{1}$. Cell1 and Cell2 have a similar structure as $R_{1}$, namely many small pores and only a few large pores. The wall-size distribution shows similar traits: there are many thin walls, but only a few thick walls. The large pores in Cell1 and Cell2 are particularly just beneath the surface, leading to blistering, as shown in Fig. 2 and the sensorial evaluation (see Table 5).

Cell3a and Cell3b show many large pores, a few smaller ones, combined with thick walls. Cell4 has slightly more large pores than Cell1 and Cell2, but the wall size distribution is quite similar.

The pore size distribution of Filler 4 is quite different from the other Fillers, it is showing more large pores and less small pores. Also in the wall size distribution we observe a higher contribution of thick walls. The pore and wall size distribution of Filler 4 is quite comparable to that of reference $R_{2}$.

\section{DSC results}

The results of analysed samples are shown in Tables S.4S.6 in the Supplementary Materials. The observed peak temperatures of the pellets are comparable to those observed for the hydrated mixes. The peak temperatures are equal to the gelatinization temperatures of potato and tapioca starches at excess water (70-80 degrees). The values of the enthalpies are not that consistent. For the wetted mixes,
Fig. 6 Cross section of XRT analysed sections of the Cell formulations

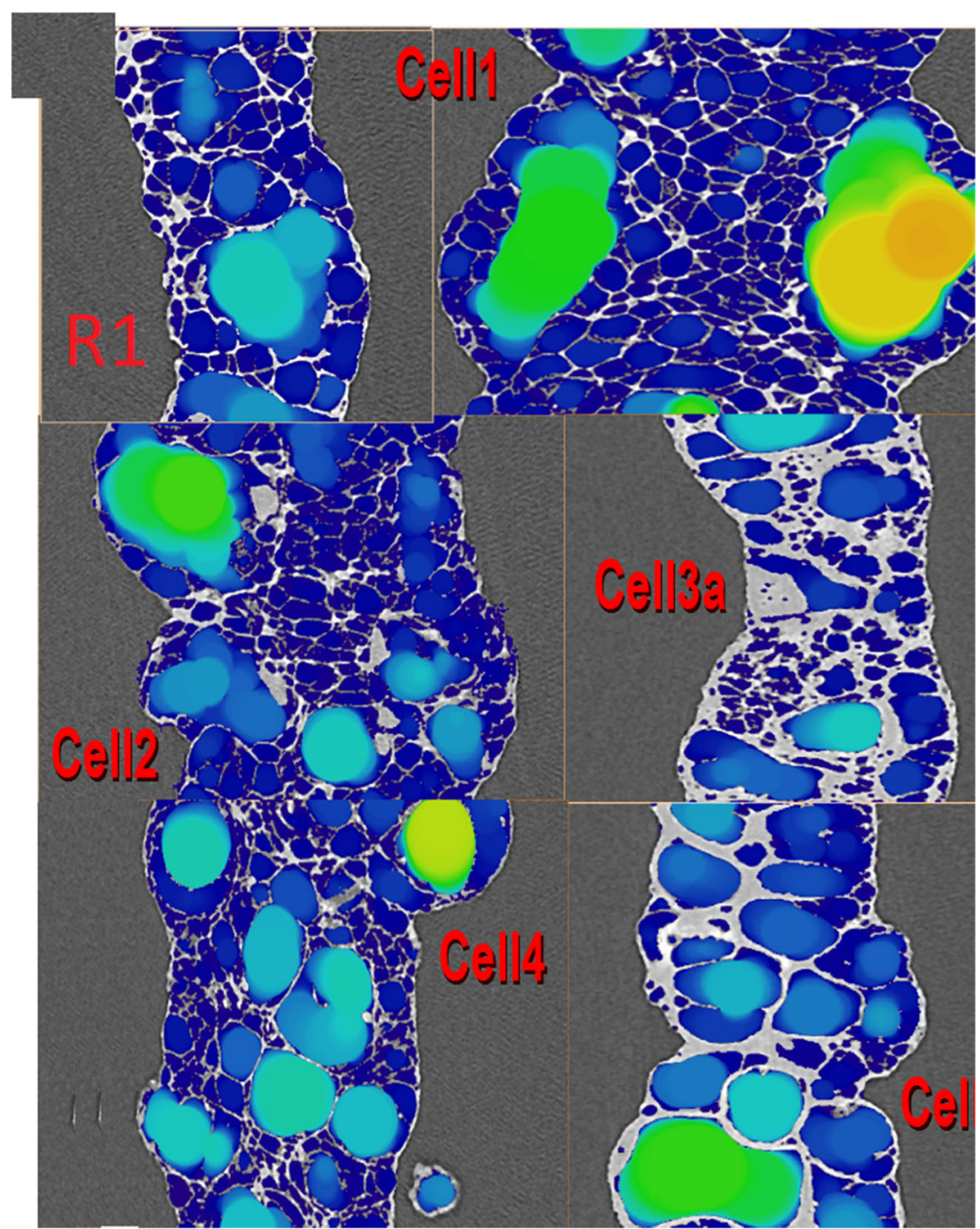


Fig. 7 Cross section of XRT analysed sections of the Filler formulations

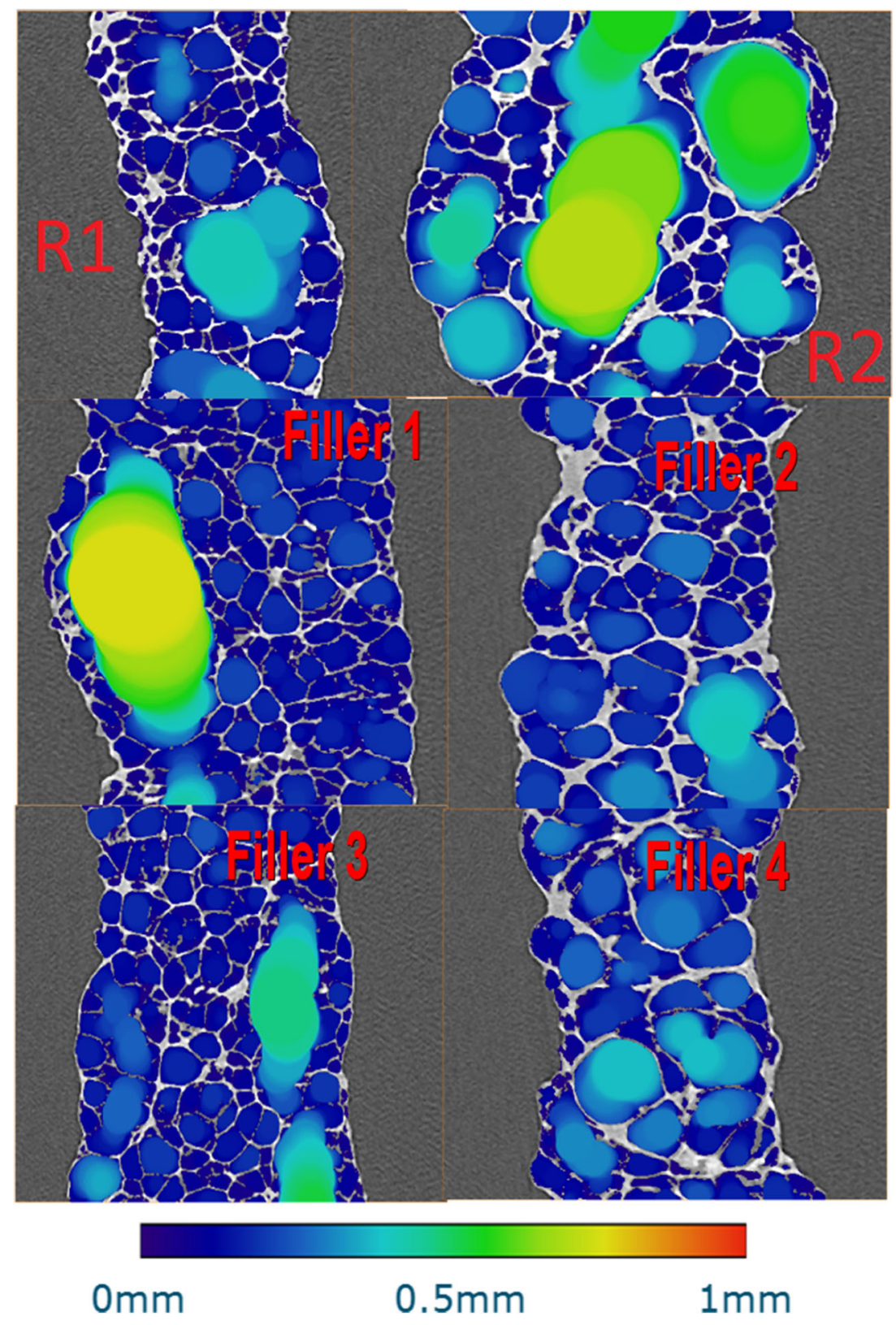

Fig. 8 Pore Size distributions of Cell (left) and Filler (right) formulations

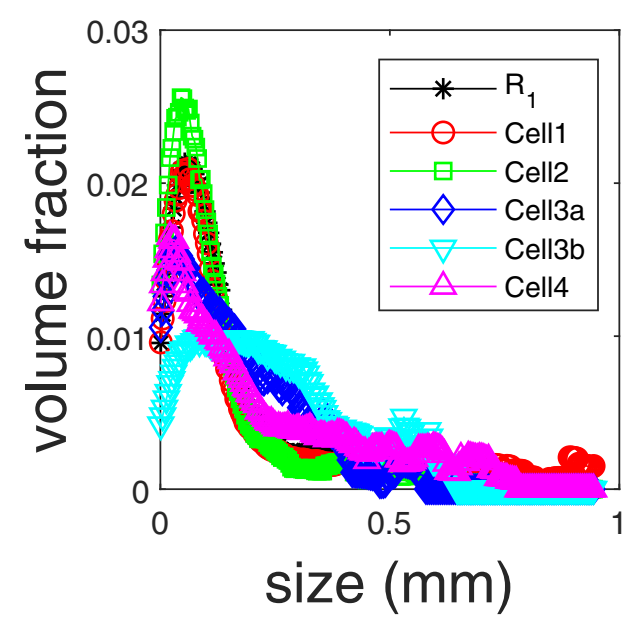


Fig. 9 Wall thickness distributions of Cell (left) and Filler (right) formulations
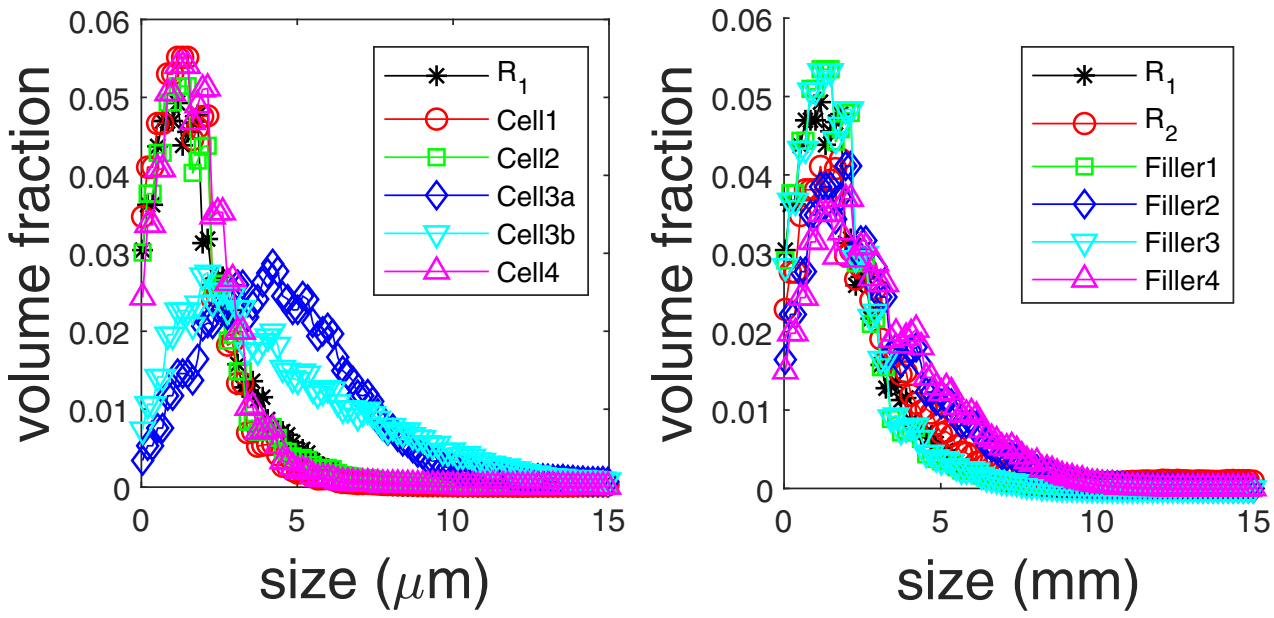

we have found $\Delta H_{\text {dough }} \approx 3 \mathrm{~J} / \mathrm{g}$, while for the wetted pellets we have found higher values: $\Delta H \approx 6 . .9 \mathrm{~J} / \mathrm{g}$ for the Cell formulations, while the Filler formulations $\Delta H \approx 3 \mathrm{~J} / \mathrm{g}$. This increase of enthalpy can be explained by enhanced swelling of starches after extrusion. In the dough, the swelling might be constrained by the cell walls, while in the extruded pellets the strength of the cell walls is lowered. This enhanced swelling of potato dehydrates (PPG and PFL) is also observed in the PSD measurements, see Fig. 5. Anyway, because of the large enthalpies, we can still conclude that the starches have not (fully) gelatinized after extrusion.

After frying, we observe that most of the formulations are fully gelatinized, as shown by the absence of a DSC peak. Only formulations containing aggregates with NTS and/or PFB $\left(R_{2}\right.$, Cell2-Cell4) still show a large enthalpy indicating a poor gelatinization due to encapsulation of the starch granules in the aggregate. We must note that XRD has registered the loss of crystallinity for all samples. However, still, energy is absorbed by starch granules during DSC, indicating incomplete gelatinization.

\section{Discussion}

Here, we first discuss the transformations of ingredients per processing step, as follows from the above presented results. We start with the hydration and mixing of dry ingredients into a dough, and subsequently, we discuss extrusion and frying. Subsequently, our research questions will be addressed, together with other learnings about ingredient functionality from the comparison of our results with the literature. Based on these discussions, we will refine our hypotheses as formulated in CDS, as shown in Table 3.

During mixing not all ingredient mix and hydrate well, as shown by the microscopy images of dry and hydrated pellets. These images show that potato cell wall material (PFB and PFL) tends to aggregate and to capture granules of native starch in the network of fibers. We have observed the strongest aggregation between PFB and NTS, which is visible in the images of the dry pellets of Cell3a/b. Cell3b formulation is extruded at a higher temperature, showing better dispersion of the aggregates, but the dispersion is still not homogeneous. Similar aggregates between PFB and NTS are also observed in the micrographs of Cell4, albeit in a smaller amount. A quick literature search shows that hydration and mixing are poorly investigated for food products [10]. However, our investigations do indicate that this is a critical step, worthy of further detailed study. Poor non-uniform hydration is expected to lead to an inhomogeneous bubble distribution [10]. Below, we discuss the aspect of aggregation further in the context of the research questions.

The results of our various analysis methods show that during the extrusion step the starchy ingredients undergo little transformations, as intended with the mild extrusion conditions. XRD and DSC show that much of the crystallinity of the native starch granules are preserved. Furthermore, the PSD results show that all particulate ingredients retain this characteristic, albeit that potato dehydrates have slightly shrunk (due to moisture removal during drying), while the native starch granules have swollen minutely.

The frying of the dry pellets makes the final expanded product, with a major transformation of the native starch granules. This is indicated by the XRD results showing the loss of crystallinity for all native starches. However, from the PSD and the micrographs of the hydrated expanded snacks we observe that many of the granules have retained their particulate shape. Again, the potato dehydrates have shrunk further, and the native starches are more swollen. The gelatinized starches might not have swollen to their full extent, due to the limited amount of water. This is shown 
by the results of the centrifugation measurements after enzymatic hydrolysis, showing that only $40 \%$ of the native potato starch granules have been hydrolysed (see Table S.3 in the Supplementary Materials). The DSC analysis of hydrated pellets and snacks shows that after frying the native starches in most formulations are fully gelatinized, except those containing aggregates containing NTS. The loss of crystallinity by the native starches is likely quite important during the expansion: the molten starch granules will instantly absorb moisture, which lowers the water activity and change the glass transition due to redistribution of water, from pre-gelatinized starch (also present in PPG and PFL) to the molten starch granules.

Sensory evaluation, microscopy (see Supplementary Materials), and XRT show that we have obtained a wide variety of texture/structure for the different formulations after the expansion of the pellets during frying. The differences amongst the Filler formulations are smaller than amongst the Cell formulations. Moreover, the texture and crispiness of the Filler formulations are quite similar to the reference $R_{1}$. It holds in general that the results of the Filler formulations are similar to $R_{1}$, as shown by the results of XRD, PSD, DSC, and water activity. Only, the XRT analysis shows there are differences between wall thickness and pore size distributions: Filler2 and Filler4 have a larger bubble wall thickness, while Filler4 also has larger bubble sizes. Nevertheless, these variations in microstructure give only slight variations in texture perception.

Given the small differences in results between Filler formulations, we can answer research questions 5-7. Due to the absence of hard fillers in formulations of Filler1 and Filler4, one can conclude that hard fillers at an amount of $8 \%$ do not contribute significantly to textural properties like crispiness or crunchiness. The size of the filler is then also not relevant for texture. In our previous study, we have observed the influence of hard fillers on texture, leading to coarse texture with large bubble sizes, albeit at larger levels of hard fillers of 20-30\%.

Gel former functionality is thought to be required during and directly after extrusion to let the pellet hold shape [27], as is also indicated in our hypotheses formulated in CDS (Table 3). If native starches do not gelatinize during extrusion, their amylose will not leach out. Hence, the native tuber and cereal starches will not deliver gel former functionality during extrusion. It can only be provided by explicitly added pre-gelatinized starches. There are several formulations without pre-gelatinized starches, namely $R_{1}$, Filler2, and Filler3. Their texture is not very different from Filler formulations with the explicit addition of pregelatinized starch, Filler1, and Filler4. $R_{1}$ only has potato granulates (PPG) as a possible source of gel former, but it is shown that potato granulates are still intact cells, without leached amylose [2]. Hence, we conclude that it is likely that in potato snacks there is no need for an explicit added gel former. Probably, the potato dough, as used in $R_{1}$, has already sufficient cohesion for its keeping shape after extrusion. This is shown by our photography of pellets and expanded snacks show there was no problem with shaping the samples.

Gel formers are said to be required for snacks, made from potato flakes, that are produced via sheeting and frying [15]. Potato dough requires sufficient strength to be sheeted, which is provided by the amylose leached from the flakes. However, too strong amylose network resists expansion, resulting in a hard texture. Yet, expanded snacks can be produced from purely waxy starch via extrusion and microwave heating [1]. It is assumed that extrusion has molten the waxy starch. After extrusion, the pellets are dried and subsequently expanded via microwave heating. Similar results are obtained with foams made from waxy potato starch (made without extrusion) [20]. We assume that the molten waxy starch matrix provides the dried pellet sufficient cohesion for expansion. The high viscosity of the starch matrix provided by the hydrogen bonding is probably sufficient to hold the expanding steam bubbles in the matrix [28]. Hence, we conclude that for the expansion stage there is no specific need for gel formers like amylose, albeit it can be used for modulation of texture.

The differences in texture perception between the Cell formulations and the references can largely be explained by the absence of potato dehydrates. In Cell1, Cell2, and Cell4 there was no PPG or PFL present, but it was mimicked with potato fibers (PFB) and pregelatinized starch (GPS or GTS). These formulations scored particularly low in crispiness, crunchiness, and hardness, but they were scored as rather gummy. Hence, it is clear that potato dehydrates can not be mimicked with just a mixture of potato fibers and gelatinized starch. The gelatinized starch has to be encapsulated by the cell wall material to render the crispiness and crunchiness as delivered by the references $R_{1}$ and $R_{2} . R_{1}$ contains only PPG, whose cells still have their integrity after extrusion. $R_{2}$ contains potato flakes (PFL), whose cells may have lost their integrity during drum drying. However, recent research has shown that the cellular matrix of potato flakes still limits the swelling of flakes and the release of starch [14]. Hence, we conclude that potato flakes in $R_{2}$ still have sufficient encapsulating functionality for rendering the desired crispiness and crunchiness. In Cell2 there is some amount of potato flakes (20\%), but the combination with a high amount of pregelatinized starch still rendered a soft and gummy texture. Given the formulations and sensory score, we assume that the soft and gummy texture is obtained if there is a large amount (> 20\%) of free pre-gelatinized starch in the matrix. Direct expanded snacks made purely from potato dehydrates have been investigated earlier [2]. Snacks made from pastes of 
Table 6 Novel hypotheses on the evolution of structure during processing of potato snacks described with CDS formalism

\begin{tabular}{ll}
\hline Step & Structure in CDS \\
\hline Dry mix & $\mathcal{W}+\mathcal{F}+\mathcal{C}+\mathcal{S}_{x}$ \\
Mixing/Extrusion & $\rightarrow\left(\mathcal{F}+\mathcal{S}_{x}\right) /(W / C)$ \\
Gelatinization & $\rightarrow(F+W / S) /(W / C)$ \\
Nucleation & $\rightarrow(F+V / S) /(V / C)$ \\
Expansion & $\rightarrow \mathcal{V} /(F /(C \| S)$ \\
Setting & $\rightarrow \mathcal{A} /\left(\mathcal{F} /\left(\mathcal{C} \| \mathcal{S}_{g}\right)\right.$ \\
\hline
\end{tabular}

pure potato granulates (PPG) were very fragile, as the granules were hardly effected by extrusion. Snacks made from potato flakes (PFL) did show good cohesion, as well as snacks made from a blend of PPG, PFL, and NPS. These findings combined with our results with $R_{1}$ and $R_{2}$, make us conclude that for formulations with PPG addition of native starch like NPS is required for good cohesion of the expanding dough. For formulations with PFL, it is not a requirement, but its addition will provide a similar texture as $R_{1}$.
The studies of [1, 2] and [20] also learns us something about nucleation. Formerly, it is assumed that nucleation occurs at the hilum of the starch granule. But, solid foams are also produced in snacks containing only potato dehydrates [2], as well as in snacks containing only native starch [1, 20]. Hence, we conclude that nucleation can occur in both (gelatinized) starch granules as well as potato dehydrates.

The similar results between Cell2 and Cell4 in the sensorial evaluation and the XRT analysis, indicate that that native or pregelatinized potato-starch can be replaced by tapioca starch. But, as said above, the tapioca granules have a strong tendency to form aggregates with potato flakes. Aggregation of potato cell walls and starch granules has been observed earlier [24]. Also, this is found in case of tapioca starch [18, 22]. It is found that the liberation of native starch granules from pulp is much more difficult for tapioca than for potato, indicating there is likely a large difference in their binding between starch granule and cell wall material.

Also for apple pomace and corn starch granules, there is thought to be some physical interaction, reducing its
Fig. 10 Schematic representation of (transformed) ingredients used in formulations
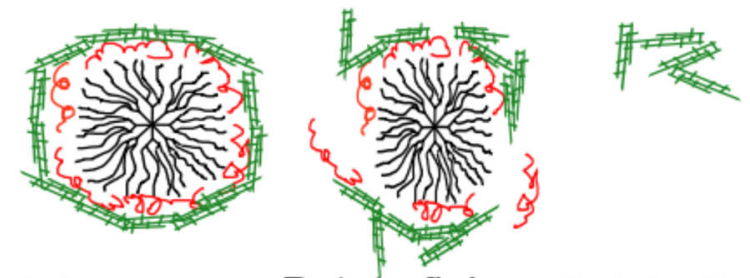

Potato puree Potato flake Potato fiber
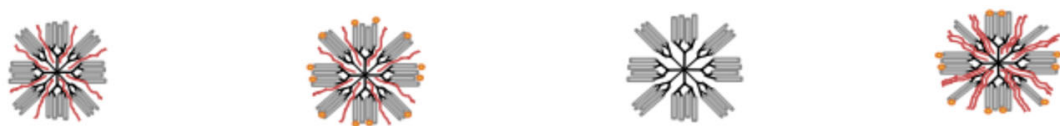

Native tuber

Native cereal

Waxy Starch

Hi-Amylo cereal
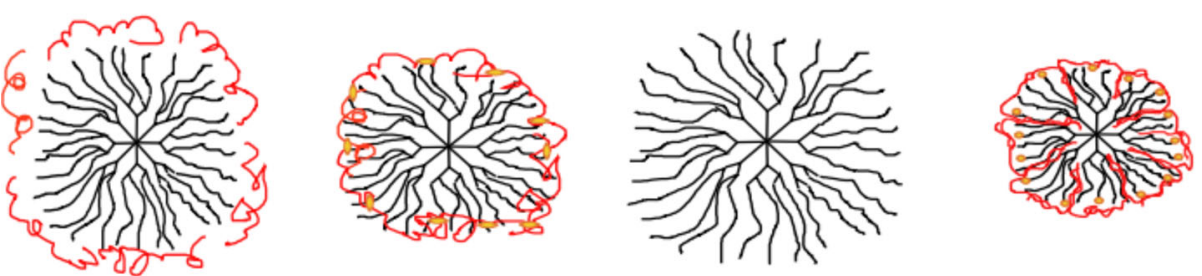

Gelled tuber
Gelled waxy

Gelled Hi-Amylo
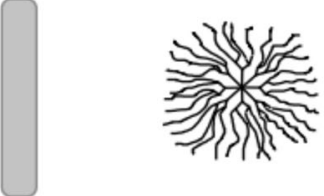

Crystal Amylopectin Amylose

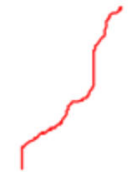

Protein/Lipid
Cell wall Fiber 
conversion during extrusion [10]. Hence, we conclude that in general there is some physical interaction between cell wall materials and starch granules, but the interaction can depend on the botanical source of the materials. Also, the interaction found between (gelatinized) starch and bacterial cellulose hints at the possible physical interaction between potato cell wall material and starch (granules) [5].

Following the above discussion, we have revisited our earlier hypotheses on structure evolution, as described with
CDS in Table 3. The novel hypotheses, again formulated in CDS, are shown in Table 6. Because of the novelty of the CDS formalism, we have also depicted our hypotheses on structure visually in Figs. 10, 11 and 12.

Above, we have concluded that there is no ingredient in the tested formulations having a gel former functionality. Hence, this functionality is to be deleted from the earlier CDS hypotheses. We have made new CDS hypotheses, which are presented in Table 6. As hard fillers and native
Fig. 11 Visual representation of CDS of the expansion of formulation $R_{1}$, upto the nucleation step

$$
W+\mathcal{F}+\mathcal{C}+\mathcal{S}_{x}
$$
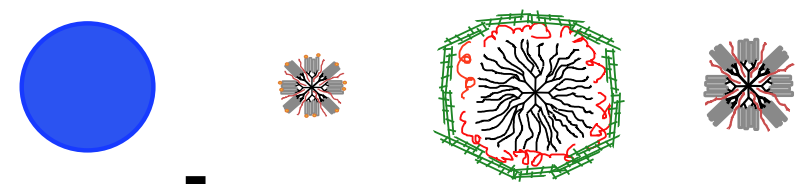

$$
\left(\mathcal{F}+S_{B}\right] /(W / C)
$$
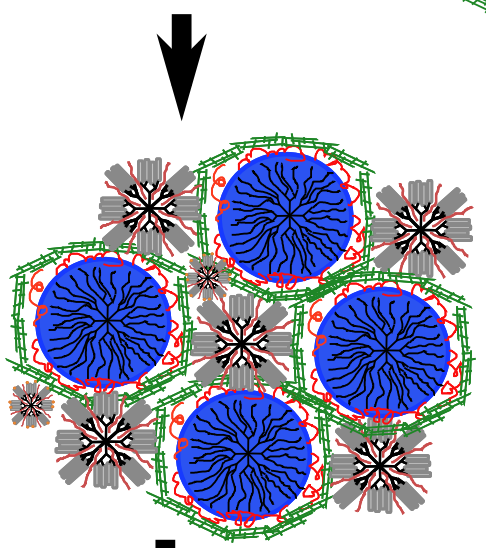

\section{Gelatinization}

$$
(J+W / S) /(W / S)
$$

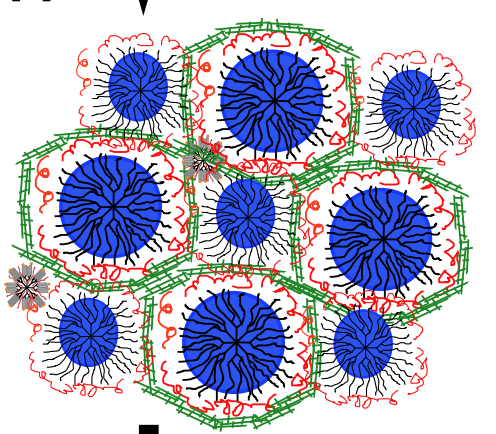

\section{Nucleation}

$$
(F+V / S) /(V / C)
$$

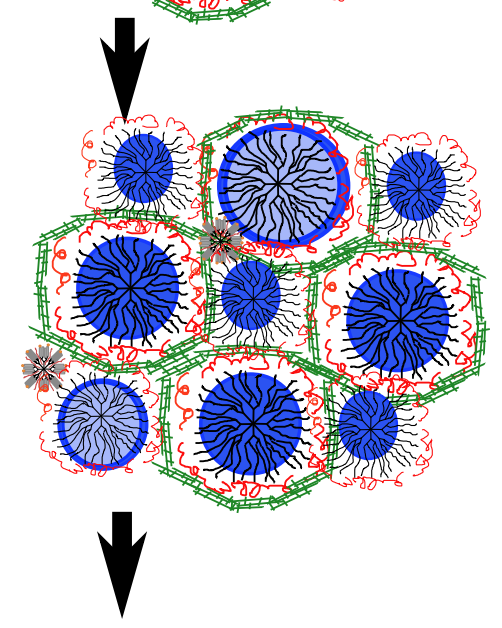




\section{Expansion}

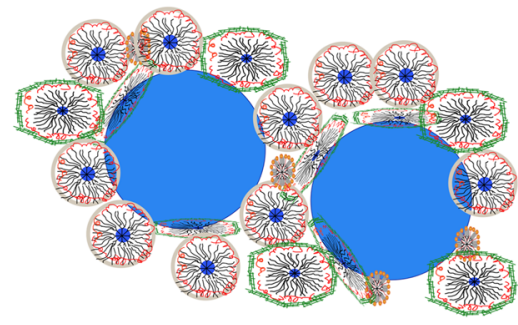

\section{Setting}

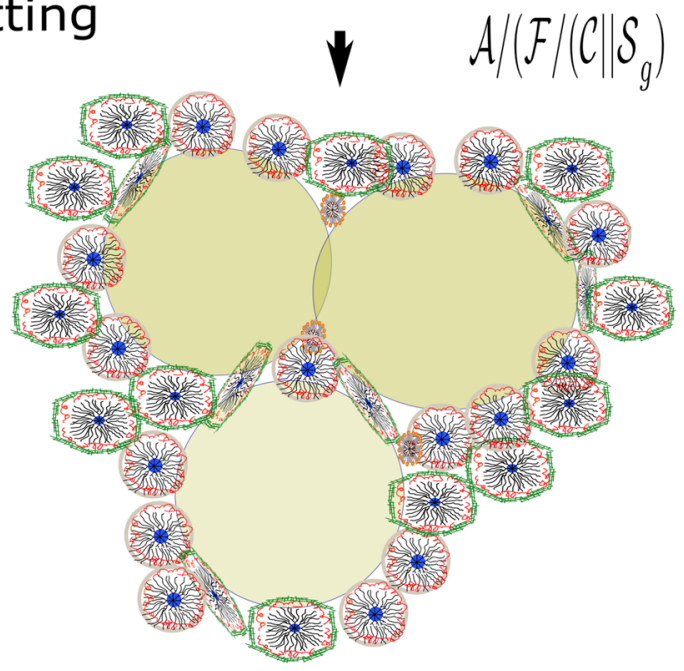

Fig. 12 Visual representation of CDS of the transformations during expansion and rupturing for formulation $R_{1}$

starches remain particulate after extrusion, the matrix of the extruded pellets must be formed by the potato dehydrates $(\mathcal{C})$. Also, they are the only ingredient that can have absorbed the water. Hence the structure of the snack pellet after extrusion is $\left(\mathcal{F}+\mathcal{S}_{x}\right) /(\mathcal{W} / \mathcal{C})$, meaning 'hard fillers and native starch dispersed in hydrated potato dehydrates'.

During frying of the pellet, first, the native starch gelatinizes at a temperature below the boiling point. The native starch loses its crystalline structure and absorbs some of the water from the matrix: $(F+W / S) /(W / C)$. Subsequently, steam bubbles nucleate either in the gelatinized starch, or the potato dehydrate: $(F+V / S) /(V / C)$. During expansion, the steam bubble grows, and is surrounded by the matrix composing of a bicontinuous structure of potato dehydrates and gelatinized starch, with some hard fillers dispersed in it: $\mathcal{V} /(F /(C \| S)$. After the snacks are taken out of the frying oil, air replaces the vapour resulting in the final structure: $\mathcal{A} /\left(\mathcal{F} /\left(\mathcal{C} \| \mathcal{S}_{g}\right)\right.$, with the starch gone into the glassy state.

In the Supplementary Material, we have expanded the CDS hypotheses for all investigated formulations, where we have also introduced the more detailed structure of the functional ingredients, as listed in Table 1. For most of the formulations, the detailed CDS hypotheses follow the general scheme presented in Table 6. Only the Cell3 formulation does not conform to the general scheme, due to the strong aggregation between potato dehydrate and the native tapioca starch. In that case, we view the matrix not as a bicontinuous structure, but as a single matrix with starch (ghost) granules dispersed in a matrix of potato flakes.

As we pointed out in the above discussion, the amount of hard fillers used in the analysed formulations did not give rise to much functionality regarding texture. Hence, they might as well be left out of the formulation. This is also shown in the CDS structure of Filler1 and Filler4, where the hard filler particles are replaced by gelatinized starches. These filler particles can hardly be distinguished from the gelatinized starch phase (originating from the tuber starch granules) of the bicontinuous matrix.

We view that to arrive at the desired crispiness of potatobased snacks it is essential to have a bicontinuous matrix of potato dehydrates and (gelatinized) potato starches. Regarding the replacement of these ingredients by nonpotato sources the native potato starch can be replaced by other tuber starches, if measures are taken for possible aggregation between potato dehydrates and tuber starches. At the moment there are no commercially available ingredients that have similar functionality as potato dehydrates. In scientific literature possible candidates have been studied, like flours from sweet potato, tapioca (cassava), or yams - still having some intact cells $[9,11,16]$. For cassava, detoxification might still be an issue [13].

\section{Conclusions}

Our analysis of the fate of starchy ingredients during processing for various reformulated snacks shows that ingredients undergo little transformations during cold extrusion. However, during frying potato and tapioca starch fully gelatinize, while cereal starches show little gelatinization and swelling. This agrees with our previous paper on this topic, where we have shown that native potato starches are soft fillers, while cereal starches are hard fillers. Despite the gelatinization of tuber starches, the particulate character of these soft fillers is retained after frying, which also holds for potato dehydrates, the other soft filler present in the formulation.

Via varying the formulation of the potato-based snack we have shown that the hard filler does not have a functionality concerning texture. Via analysis of the different structures of the snack formulations with the CDS formalism, we conclude that for the desired crunchiness and crispiness it is required that the matrix is composed of a bicontinuous structure of two soft fillers: gelatinized starches and potato dehydrates, with the latter having a significant portion of intact cells. 
Regarding ingredient replacement we conclude that the gelatinized potato starch can be replaced by other tuber starches if aggregation with cell wall materials can be prevented (via either intactness of potato cells or extrusion conditions). Potato dehydrates might be replaced by flours of other tuber starches, having intact cells. But currently, flakes of alternative tuber roots are not commercially available.

Acknowledgments The authors would like to acknowledge the funding made available from PepsiCo which enabled this work. The views expressed in this report are those of the authors and do not necessarily represent the position or policy of PepsiCo, Inc.

Open Access This article is licensed under a Creative Commons Attribution 4.0 International License, which permits use, sharing, adaptation, distribution and reproduction in any medium or format, as long as you give appropriate credit to the original author(s) and the source, provide a link to the Creative Commons licence, and indicate if changes were made. The images or other third party material in this article are included in the article's Creative Commons licence, unless indicated otherwise in a credit line to the material. If material is not included in the article's Creative Commons licence and your intended use is not permitted by statutory regulation or exceeds the permitted use, you will need to obtain permission directly from the copyright holder. To view a copy of this licence, visit http:// creativecommonshorg/licenses/by/4.0/.

\section{References}

1. C. Boischot, C.I. Moraru, Kokini. JL, Factors that influence the microwave expansion of glassy amylopectin extrudates. Cereal Chem. 80(1), 56-61 (2003)

2. A. Cheyne, J. Barnes, S. Gedney, D. Wilson, Extrusion Behaviour of cohesive potato starch pastes Ii. microstructure-process interactions. J. Food Eng. 66(1), 13-24 (2005)

3. C. Cunin, S. Handschin, P. Walther, F. Escher, Structural changes of starch during cooking of durum wheat pasta. LWT-Food Sci. Technol. 28(3), 323-328 (1995)

4. M.R. Debet, M.J. Gidley, Three Classes of starch granule swelling Influence of surface proteins and lipids. Carbohydr. Polym. 64(3), 452-465 (2006)

5. P. Díaz-Calderón, B. MacNaughtan, S. Hill, T. Foster, J. Enrione, J. Mitchell, Changes in gelatinisation and pasting properties of various starches (wheat, maize and waxy maize) by the addition of bacterial cellulose fibrils. Food Hydrocolloids 80, 274-280 (2018)

6. J. Fan, J.R. Mitchell, J.V.M. Blanshard, A computer simulation of the dynamics of bubble growth and shrinkage during extrudate expansion. J. Food Eng. 23(3), 337-356 (1994)

7. Y. Fukai, E. Takaki, S. Kobayashi, Characteristic change of rice starch granules by enzymatic treatment. J. Jpn. Soc. Starch Sci. 40(3), 263-269 (1993)

8. R.C.E. Guy, Raw materials for extrusion cooking processes. The technology of extrusion cooking, 52-72 (1994)

9. J. He, L. Cheng, Z. Gu, Y. Hong, Z. Li, Effects of low-temperature blanching on tissue firmness and cell wall strengthening during sweet potato flour processing. Int. J. Food Sci. Technol. 49(5), 1360-1366 (2014)
10. E.L. Karkle, L. Keller, H. Dogan, S. Alavi, Matrix transformation in fiber-added extruded products: impact of different hydration regimens on texture, microstructure and digestibility. J. Food Eng. 108(1), 171-182 (2012)

11. A.G. Konan, J. Brunnschweiler-Beez, J. Nuessli-Guth, F. Escher, B. Conde-Petit, Texture and microstructure characterization of pastes reconstituted from drum-dried flakes of yam (dioscorea spp.). Eur. Sci. J. 10(3) (2014)

12. A. Lopez-Rubio, B.M. Flanagan, E.P. Gilbert, M.J. Gidley, A novel approach for calculating starch crystallinity and its correlation with double helix content: A combined xrd and nmr study. Biopolymers 89(9), 761-768 (2008)

13. A. Panghal, C. Munezero, P. Sharma, N. Chhikara, Cassava toxicity, detoxification and its food applications: a review. Toxin Rev. 1-16 (2019)

14. S. Reyniers, N. De Brier, S. Matthijs, K. Brijs, J.A. Delcour, Impact of physical and enzymatic cell wall opening on the release of pre-gelatinized starch and viscosity forming potential of potato flakes. Carbohydr. Polym. 194, 401-410 (2018)

15. S. Reyniers, N. Vluymans, N. De Brier, N. Ooms, S. Matthijs, K. Brijs, J.A. Delcour, Amylolysis as a tool to control amylose chain length and to tailor gel formation during potato-based crisp making. Food Hydrocoll. 103, 105658 (2020)

16. E. Rodríguez-Sandoval, A. Fernández-Quintero, G. Cuvelier, Stress relaxation of reconstituted cassava dough. LWT-Food Sci. Technol. 42(1), 202-206 (2009)

17. M. Saeleaw, G. Schleining, Effect of blending cassava starch, rice, waxy rice and wheat flour on physico-chemical properties of flour mixtures and mechanical and sound emission properties of cassava crackers. J. Food Eng. 100(1), 12-24 (2010)

18. K. Saengchan, M. Nopharatana, R. Lerdlattaporn, W. Songkasiri, Enhancement of starch-pulp separation in centrifugal-filtration process Effects of particle size and variety of cassava root on free starch granule separation. Food Bioprod. Process. 95, 208-217 (2015)

19. E. Sarikaya, T. Higasa, M. Adachi, B. Mikami, Comparison of degradation abilities of $\alpha$-and $\beta$-amylases on raw starch granules. Process Biochem. 35(7), 711-715 (2000)

20. M. Sjöqvist, P. Gatenholm, Effect of water content in potato amylopectin starch on microwave foaming process. J. Polym. Environ. 15(1), 43 (2007)

21. S. Srichuwong, T.C. Sunarti, T. Mishima, N. Isono, M. Hisamatsu, Starches from different botanical sources ii Contribution of starch structure to swelling and pasting properties. Carbohydr. Polym. 62(1), 25-34 (2005)

22. K. Sriroth, R. Chollakup, S. Chotineeranat, K. Piyachomkwan, C.G. Oates, Processing of cassava waste for improved biomass utilization. Bioresour. Technol. 71(1), 63-69 (2000)

23. H. This, Modelling dishes and exploring culinary 'precisions': the two issues of molecular gastronomy. British J. Nutrit. 93(S1), S139-S146 (2005)

24. J. Tian, S. Chen, H. Zhang, H. Fang, Y. Sun, D. Liu, R.J. Linhart, $\mathrm{X}$. Ye, Existing cell wall fragments modify the thermal properties and hydrolysis of potato starch. Food Hydrocoll. 85, 229-232 (2018)

25. R.G.M. van der Sman, Filler functionality in edible solid foams. Adv. Colloid Interface Sci. 231, 23-35 (2016)

26. R.G.M. van der Sman, J.R. Bows, Critical factors in microwave expansion of starchy snacks. J. Food Eng. 211, 69-84 (2017)

27. R.G.M. van der Sman, J. Broeze, Structuring of indirectly expanded snacks based on potato ingredients A review. J. Food Eng. 114(4), 413-425 (2013)

28. R.G.M. van der Sman, J. Broeze, Multiscale analysis of structure development in expanded starch snacks. J. Phys. Condens. Matter 26(46), 464103 (2014) 
29. R.G.M. van der Sman, S. Renzetti, Understanding functionality of sucrose in biscuits for reformulation purposes. Crit. Rev. Food Sci. Nutrit. 59(14), 2225-2239 (2019)

30. R.G.M. van der Sman, S. Renzetti, Understanding functionality of sucrose in cake for reformulation purposes Critical reviews in food science and nutrition. https://doi.org/10.1080/10408398.2020.178 6003 (2020)

31. R.G.M. van der Sman, A.J. van der Goot, The science of food structuring. Soft Matter 5(3), 501-510 (2009)
32. R.G.M. van der Sman, H.M. Vollebregt, M.B.J. Meinders, A. Beri, Effects of filler ingredients on the structure and texture of starchy, extruded snacks. Food Struct. 18, 1-13 (2018)

33. C. Zweifel, B. Conde-Petit, F. Escher, Thermal modifications of starch during high-temperature drying of pasta. Cereal Chem. 77(5), 645-651 (2000)

Publisher's Note Springer Nature remains neutral with regard to jurisdictional claims in published maps and institutional affiliations. 